University of Wollongong

Research Online

Australian Institute for Innovative Materials -

Papers

Australian Institute for Innovative Materials

$1-1-2013$

\title{
Mechanical properties of interpenetrating polymer network hydrogels based on hybrid ionically and covalently crosslinked networks
}

Sina Naficy

University of Wollongong, snaficy@uow.edu.au

Shota Kawakami

Kyushu Institute of Technology

Sasha Sadeghovaad

University of Wollongong

Minato Wakisaka

Kyushu Institute of Technology

Geoffrey M. Spinks

University of Wollongong, gspinks@uow.edu.au

Follow this and additional works at: https://ro.uow.edu.au/aiimpapers

Part of the Engineering Commons, and the Physical Sciences and Mathematics Commons

Research Online is the open access institutional repository for the University of Wollongong. For further information contact the UOW Library: research-pubs@uow.edu.au 


\title{
Mechanical properties of interpenetrating polymer network hydrogels based on hybrid ionically and covalently crosslinked networks
}

\author{
Abstract \\ Hydrogels are polymer networks swollen in water. Because of their soft and wet nature, and their ability to \\ show large volume changes, hydrogels can be useful in many biomedical and actuator applications. In \\ these applications, it is crucial to tune the mechanical and physical properties of a hydrogel in a \\ controllable manner. Here, interpenetrating polymer networks (IPNs) made of a covalently crosslinked \\ network and an ionically crosslinked network were produced to investigate the effective parameters that \\ control the physical and mechanical properties of an IPN hydrogel. Covalently crosslinked polyacrylamide \\ (PAAm) or poly(acrylic acid) (PAA) networks were produced in the presence of alginate (Alg) that was \\ then ionically crosslinked to produce the IPN hydrogels. The effect of ionic crosslinking, degree of \\ covalent crosslinking, AAm : Alg and AA : Alg ratio on the swelling ratio, tensile properties, indentation \\ modulus, and fracture energy of IPN hydrogels was studied. A hollow cylindrical hydrogel with gradient \\ mechanical properties along its length was developed based on the obtained results. The middle section \\ of this hydrogel was designed as a $\mathrm{pH}$ triggered artificial muscle, while each end was formulated to be \\ harder, tougher, and insensitive to $\mathrm{pH}$ so as to function as a tendonlike material securing the gel muscle to \\ its mechanical supports.

\section{Keywords} \\ polymer, interpenetrating, properties, mechanical, network, ionically, networks, hybrid, hydrogels, \\ covalently, crosslinked

\section{Disciplines} \\ Engineering | Physical Sciences and Mathematics

\section{Publication Details} \\ Naficy, S., Kawakami, S., Sadeghovaad, S., Wakisaka, M. \& Spinks, G. Maxwell. (2013). Mechanical \\ properties of interpenetrating polymer network hydrogels based on hybrid ionically and covalently \\ crosslinked networks. Journal of Applied Polymer Science, 130 (4), 2504-2513.
}




\title{
Mechanical Properties of Interpenetrating Polymer Network Hydrogels Based on Hybrid Ionically and Covalently Crosslinked Networks
}

\author{
Sina Naficy ${ }^{1}$, Shota Kawakami ${ }^{2}$, Sasha Sadegholvaad ${ }^{1}$, Minato Wakisaka ${ }^{2}$ and Geoffrey M. \\ Spinks ${ }^{1}$ \\ ${ }^{1}$ ARC Centre of Excellence in Electromaterials Science and Intelligent Polymer Research Institute, \\ AIIM Faculty, Innovation Campus, University of Wollongong, Squires Way, North Wollongong, \\ NSW, 2522, Australia. \\ ${ }^{2}$ Graduate School of Life Science and Systems Engineering, Kyushu Institute of Technology, 2-4 \\ Hibikino, Wakamatsu-ku, Kitakyushu, 808-0196, Japan. \\ Correspondence to: Geoffrey Spinks (E-mail: gspinks@uow.edu.au )
}

\begin{abstract}
Hydrogels are polymer networks swollen in water. Because of their soft and wet nature, and their ability to show large volume changes, hydrogels can be useful in many biomedical and actuator applications. In these applications it is crucial to tune the mechanical and physical properties of a hydrogel in a controllable manner. Here, interpenetrating polymer networks (IPNs) made of a covalently crosslinked network and an ionically crosslinked network were produced to investigate the effective parameters that control the physical and mechanical properties of an IPN hydrogel. Covalently crosslinked polyacrylamide (PAAm) or poly(acrylic acid) (PAA) networks were produced in the presence of alginate (Alg) that was then ionically crosslinked to produce the IPN hydrogels. The effect of ionic crosslinking, degree of covalent crosslinking, AAm:Alg and AA:Alg ratio on the swelling ratio, tensile properties, indentation modulus and fracture energy of IPN hydrogels was studied. A hollow cylindrical hydrogel with gradient mechanical properties along its length was developed based on the obtained results. The middle section of this hydrogel was designed as a $\mathrm{pH}$ triggered artificial muscle, while each end was formulated to be harder, tougher and insensitive to $\mathrm{pH}$ so as to function as a tendon-like material securing the gel muscle to its mechanical supports.
\end{abstract}

KEYWORDS Alginate, Hydrogels, Indentation, Mechanical Properties, Polyacrylamide, Swelling Ratio, Tensile. 


\section{INTRODUCTION}

Hydrogels are three-dimensional polymer networks made of hydrophilic polymer chains that are chemically or physically crosslinked. As a result of this crosslinking, hydrogels do not dissolve in water; instead, they readily swell in aqueous solutions and undergo large volume changes. Environmentally sensitive hydrogels can also be produced from hydrophilic, stimuli-responsive polymer networks that change volume in response to an external signal such as a change in temperature or chemical environment. These materials are attractive candidates for various biomedical applications and artificial muscles. ${ }^{1-5}$

Certain applications for hydrogels are limited by their insufficient mechanical strength and toughness. Conventionally produced synthetic hydrogels typically exhibit low Young's modulus (1 - $100 \mathrm{kPa})$, low tensile and compression strength $(1-100 \mathrm{kPa})$ and low fracture energy $\left(<10 \mathrm{~J} \mathrm{~m}^{-2}\right)$, while retaining high swelling ratios $(10-100){ }^{6}$ Various methods are now available to toughen hydrogels allowing their use in load-bearing applications. Amongst the methods available for producing tough hydrogels are slip-link networks, ${ }^{7}$ nanocomposite hydrogels, ${ }^{8}$ double network hydrogels, ${ }^{9}$ multifunctional crosslinked hydrogels, ${ }^{10}$ homogeneous hydrogels ${ }^{11,12}$ and hybrid ionic-covalent IPN hydrogels. ${ }^{13}$ All these methods have been shown to improve the hydrogel strength and some significantly improve hydrogel toughness.

One approach to modulate the mechanical properties of a hydrogel is to utilise an interpenetrating polymer network (IPN) system. ${ }^{14}$ An ideal IPN is a mixture of two crosslinked networks, interpenetrating within each other at the molecular level. IPNs usually show physical and mechanical properties intermediate of the constituent networks, so the blending of a less-swellable, stiffer hydrogel with a more-swellable, softer hydrogel can be used to tune the IPN modulus and swellability. The additional bonus of such IPNs is the major improvement in toughness that often results. ${ }^{15}$ Enhanced toughness is known to occur in IPNs known as "double network hydrogels"9 when the first network is more tightly crosslinked than the second network and the molar ratio of the second network to the first network is greater than $\sim 5 .{ }^{16-18} \mathrm{DN}$ hydrogels are prepared by a sequential twostep polymerisation process. Soaking the first formed network in the monomer solution of the second network causes considerable swelling so that the final gel shape and volume of the DN hydrogel are difficult to control. One-pot IPNs in which at least one of the networks is a biopolymer have been known for some time ${ }^{19-21}$ and have recently been shown to display considerable toughness. ${ }^{13,22}$ These hybrid gels are conveniently formed in a one-step synthesis by dissolving the biopolymer in a solution of the monomer that is then crosslinked to form the synthetic covalent network. The mechanical performance and physical properties of these hybrid hydrogels can be mainly tuned by the ratio of the two networks, and the degree of crosslinking in each network.

The aim of the present study was to investigate the main parameters that influence the mechanical performance of hybrid ionic-covalent IPN hydrogels. The feasibility of exploiting the IPN approach to introduce step-gradients in the hydrogel physical and mechanical properties was also investigated. Here, we used polyacrylamide (PAAm) and poly(acrylic acid) (PAA) as the covalent network and alginate as the ionic network. Alginate is a naturally occurring anionic polysaccharide which has many biomedical applications because of its biocompatibility and low toxicity. ${ }^{23}$ Since alginate is an anionic polymer, it can be ionically crosslinked using a divalent cation (e.g. $\mathrm{Ca}^{2+}$ ) to form a gel. Mechanical properties of hydrogels made of ionically crosslinked alginate vary depending on the source of alginate, concentration of alginate and the cation that is used to make the gel. ${ }^{24}$ PAAm and PAA are two commonly used water soluble polymers, and form hydrogels upon crosslinking which mainly takes place during the radical polymerization process. Unlike PAAm which is a neutral 
polymer, PAA has carboxylic acid side groups, which make the polymer $\mathrm{pH}$-sensitive with a $\mathrm{p} K_{\mathrm{a}}$ value around $4.25 .^{25}$

\section{EXPERIMENTAL}

\section{Materials}

Acrylamide (AAm, $40 \mathrm{wt} \%$ aqueous solution; Sigma-Aldrich) and acrylic acid (AA; Sigma-Aldrich) were used as received to make the covalent network. Potassium persulphate (KPS; Sigma-Aldrich) was used as the radical initiator, and $N, N^{\prime}$-methylenebisacrylamide (MBAA; Sigma-Aldrich) was used as the chemical crosslinking agent. Sodium alginate from brown algae (Alg; Sigma-Aldrich) was used to make the ionic network. Calcium chloride $\left(\mathrm{CaCl}_{2}\right.$; Sigma-Aldrich) was used for ionic crosslinking of Alg. To control the $\mathrm{pH}$ of the solutions when needed, small amount of hydrochloric acid (HCl, 0.5 M; Chem Supply, Australia) or sodium hydroxide (NaOH; Sigma-Aldrich) was used. Deionized water was used in preparation of all solutions.

\section{Hydrogel Preparation}

PAAm-Alg. AAm monomer stock solution was prepared by dissolving KPS ( $0.1 \mathrm{~mol} \%$ based on AAm) in AAm aqueous solution (40 wt\%). Various amounts of MBAA (0, 0.1, 0.2, 0.5 or $2 \mathrm{~mol} \%$ based on AAm) were added to the above solution to investigate the effect of chemical crosslinking on the physical and mechanical properties of the hydrogels. Alg was dissolved in water to make up the Alg stock solution. Adequate portions from AAm monomer stock solution and Alg stock solution along with extra water were mixed together to prepare the final AAm-Alg polymerization solution. The final concentration of monomer (i.e. AAm) was controlled to remain at $12 \mathrm{wt} \%$, with Alg concentration varied from $1 \mathrm{wt} \%$ to $4 \mathrm{wt} \%$ (1, 2, 3 or $4 \mathrm{wt} \%)$. Before polymerization, the AAm-Alg solution was degassed at room temperature. Glass slides $(50 \mathrm{~mm} \times 75 \mathrm{~mm}$, treated with octadecyltrichlorosilane (90 \%; Sigma-Aldrich), hexane and hydrogen peroxide (35\%; Ajax Finechem, Australia) to render the surface hydrophobic ${ }^{26}$ ) separated with a $1 \mathrm{~mm}$ silicon gasket were used as the polymerization mould. After degassing, the AAm-Alg polymerization solution was transferred into the mould and polymerization was carried out at $60{ }^{\circ} \mathrm{C}$ for $6 \mathrm{hrs}$ in a fan-forced oven. After polymerization, hydrogels were removed from the moulds and stored in a $2 \mathrm{wt} \% \mathrm{CaCl}_{2}$ aqueous solution for at least 3 days to ionically crosslink Alg. After this period, the hybrid hydrogels were removed from the $\mathrm{CaCl}_{2}$ solution and washed thoroughly with deionized water for several days prior to their characterization. To investigate the effect of ionic crosslinking of Alg, hydrogels were also prepared without ionic crosslinking. For these samples the as-prepared hydrogels were stored in deionized water directly after the radical polymerization with no ionic crosslinking step. Control single network hydrogels with no Alg were also made based on the above procedure with deionized water used instead of Alg stock solution.

PAA-Alg. A similar procedure was used here to make the PAA-Alg hydrogels. Briefly, AA monomer was added to the $0.1 \mathrm{M} \mathrm{NaOH}$ solution (50 wt\%) followed by adding KPS ( $0.1 \mathrm{~mol} \%$ based on AA) and MBAA ( 0.2 or $0.5 \mathrm{~mol} \%$ based on AA) to the AA solution. The Alg stock solution was prepared by dissolving Alg in the $0.1 \mathrm{M} \mathrm{NaOH}$ solution. The reason for using $0.1 \mathrm{M} \mathrm{NaOH}$ solution to make up AA monomer stock solution and Alg stock solution was that Alg was coagulated in the acidic pHs. The aqueous solution of AA is acidic and can result an inhomogeneous mixture when Alg stock 
solution is added to the acidic AA monomer solution. To avoid this inhomogeneity and obtain a clear polymerization solution $0.1 \mathrm{M} \mathrm{NaOH}$ solution was used instead of deionized water. Adequate portions of AA monomer stock solution and Alg stock solution were used to prepare the final polymerization solutions in which AA concentration was kept constant at $15 \mathrm{wt} \%$, and Alg concentration varied from $1 \mathrm{wt} \%$ to $3 \mathrm{wt} \%$ (1, 2, or $3 \mathrm{wt} \%)$. Again $0.1 \mathrm{M}$ NaoH was used when needed to adjust the composition of these AA-Alg polymerization solutions. After degassing, the AA-Alg polymerization solution was transferred into the moulds followed by the thermal polymerization at $60{ }^{\circ} \mathrm{C}$ for $6 \mathrm{hrs}$. After the polymerization, hydrogels were removed from the moulds and stored in the $2 \mathrm{wt} \% \mathrm{CaCl}_{2}$ aqueous solution for at least 3 days, followed by a thorough wash in deionized water for several days. Similar to the PAAm-Alg case, hydrogels with no ionic crosslinking were prepared by immersing the asprepared hydrogels in deionized water immediately after the polymerization. Single network PAA hydrogel was prepared as the control by replacing the Alg stock solution with $0.1 \mathrm{M} \mathrm{NaOH}$ solution in the polymerization solution preparation step.

\section{Characterization}

Tensile test. Tensile test was performed on equilibrium swollen hydrogels using an EZ-S mechanical tester (Shimadzu, Japan). Tensile samples were prepared by cutting the hydrogels into $40 \mathrm{~mm} \times 5 \mathrm{~mm}$ strips. The cross-head speed was $10 \mathrm{~mm} / \mathrm{min}$ and a $10 \mathrm{~N}$ load cell was used in all measurements. All experiments were performed at least five times on two separately made batches of hydrogels. The results presented here are the average values with standard deviations.

Indentation test. Modulus of hydrogels was measured using an indentation technique. The EZ-S mechanical tester was used in the compression mode with a $2 \mathrm{~N}$ load cell and the cross-head speed of $0.1 \mathrm{~mm} / \mathrm{min}$. A plastic head (acrylate) with a flat tip $(0.990 \mathrm{~mm}$ in diameter) was used as the indenter to apply force to the hydrogel surface. The relationship between applied force $(F)$, indentation depth $(d)$ and reduced modulus $\left(E^{*}\right)$ is: ${ }^{27}$

$$
\begin{gathered}
F=2 a E^{*} d \\
\left(E^{*}\right)^{-1}=\left(1-v_{1}^{2}\right) E_{1}^{-1}+\left(1-v_{2}^{2}\right) E_{2}^{-1}
\end{gathered}
$$

Where $a$ is the indenter radius, $E_{1}$ and $E_{2}$ are the indenter and substrate moduli, $v_{1}$ and $v_{2}$ are the Poisson's ratios of the indenter and the substrate, respectively. When the indenter is made of a material which is much stiffer than the substrate $\left(E_{1} \gg E_{2}\right)$ the first part on the right hand side of equation 2 becomes negligible. By substituting the Poisson's ratio of the swollen hydrogel substrate $v_{2}$ with 0.5 , the equations 1 and 2 reduce to:

$$
F=(8 / 3) a E_{2} d
$$

Here, $F$ and $d$ were recorded from the indentation tests and $a$ was the radius of the indenter tip (0.495 $\mathrm{mm})$. To obtain the hydrogel modulus, the applied force was plotted against indentation depth for $d \leq$ $100 \mu \mathrm{m}$. The slope of the linear fit was then used to calculate $E_{2}$ using equation 3.

Fracture test. Fracture was investigated as the propagation of a crack throughout the hydrogel at a constant speed. A trouser tear test was performed on $7.5 \mathrm{~mm} \times 50 \mathrm{~mm}$ samples with a pre-existing central cut $(30 \mathrm{~mm})$ dividing the specimens into two equal legs. The legs were then pulled apart (10 
$\mathrm{mm} / \mathrm{min}$ ) while the crack propagated down the centre of the specimen. Fracture energy $\left(G_{c}\right)$ was determined by:

$$
G_{c}=2 F / h
$$

where $F$ is applied force and $h$ is sample thickness. All experiments were repeated at least five times.

Swelling ratio. Swelling ratio was expressed as the ratio of hydrogel mass before and after drying. The mass of fully swollen hydrogels was recorded $\left(W_{S}\right)$ first, then hydrogels were dried completely at $60{ }^{\circ} \mathrm{C}$ for several days. The mass of dried samples was measured again $\left(W_{d}\right)$. The swelling ratio $(Q)$ was determined as:

$$
Q=W_{s} / W_{d}
$$

At least three repeats were carried out on samples from two separately made batches of hydrogels.

\section{RESULTS AND DISCUSSION}

\section{Swelling Ratio}

The equilibrium swelling ratios of the PAAm- and PAA-based hydrogels prepared with different Alg concentrations are shown in Figures 1 and 2. A clear difference was observed in all cases between samples prepared with and without ionic crosslinking. In ionically crosslinked PAAm-Alg and PAAAlg IPN hydrogels, a higher Alg concentration resulted in lower swelling ratios, with slightly higher swelling occurring when a smaller concentration of chemical crosslinker (MBAA) was used. The swelling ratio of PAAm hydrogels decreased from 15 - 30 (depending on the degree of chemical crosslinking) to $8-10$ as the Alg concentration was increased from 0 to $4 \mathrm{wt} \%$. A similar trend was observed for ionically crosslinked PAA-Alg IPN hydrogels (Figure 2), although much higher swelling ratio (2 - 10 times) was recorded in comparison with ionically crosslinked PAAm-Alg IPN hydrogels. The equilibrium swelling ratio of PAA-Alg IPN hydrogels decreased from 40 - 100 to 12 - 24 with increasing Alg from $0-3 \mathrm{wt} \%$. Since the $\mathrm{pH}$ of the polymerization solution was quite basic when PAA-based hydrogels were synthesized, the resulting PAA network was negatively charged. Charged polyelectrolytes generally swell more than neutral hydrogels, so it is expected that the PAA-based hydrogels swelled more than PAAm-based hydrogels.
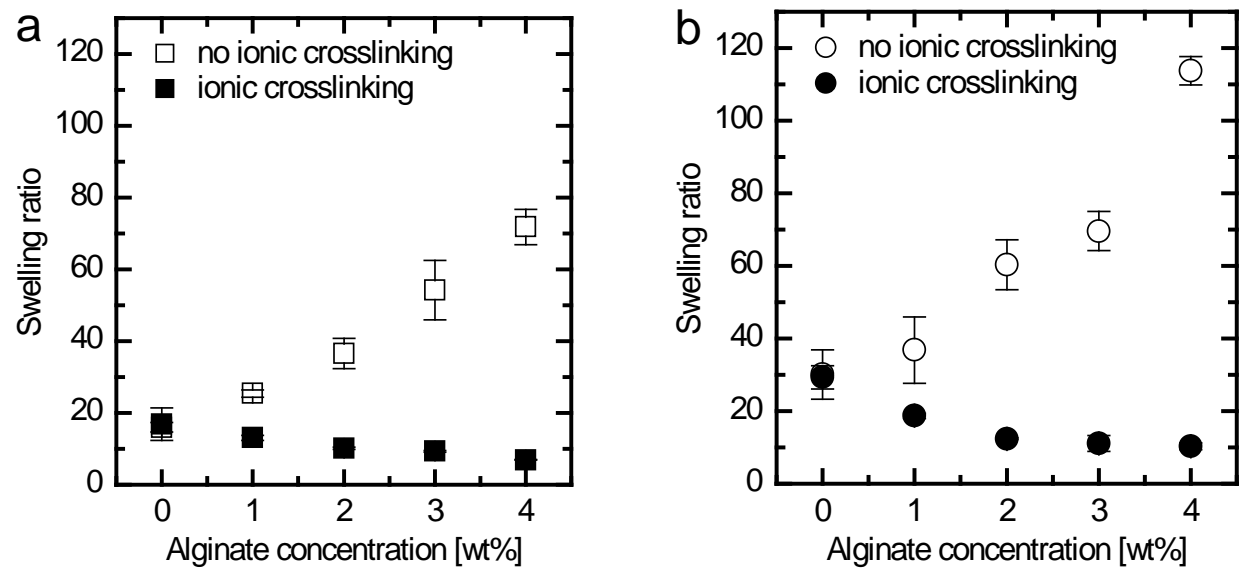
Figure 1 Equilibrium swelling ratios of PAAm-based hydrogels in deionized water as a function of Alg concentration in the polymerization solution. MBAA chemical crosslinker concentration in the polymerization solution was (a) $0.5 \mathrm{~mol} \%$ and (b) $0.2 \mathrm{~mol} \%$. Ionically crosslinked hydrogels obtained by first immersing the hydrogels in $2 \mathrm{wt} \% \mathrm{CaCl}_{2}$ solution are represented by filled symbols and hydrogels without ionic crosslinking by open symbols. In some cases the error bars are too small to be seen.
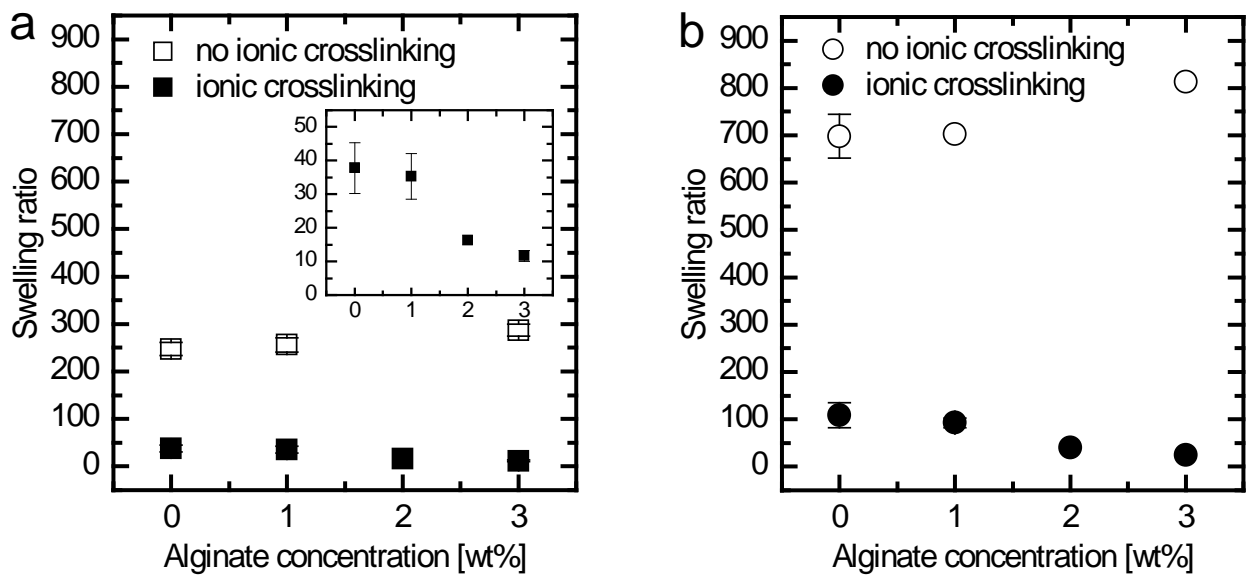

Figure 2 Equilibrium swelling ratio of PAA-based hydrogels in deionized water as a function of Alg concentration in the polymerization solution. MBAA concentration in the polymerization solution is (a) $0.5 \mathrm{~mol} \%$ and (b) $0.2 \mathrm{~mol} \%$. Ionically crosslinked hydrogels are represented by filled symbols, and hydrogels with no ionic crosslinking by open symbols. Inset in (a) magnifies the ionically crosslinked PAA-Alg hydrogels with 0.5 mol\% MBAA.

Surprisingly, the equilibrium swelling of both PAAm- and PAA-based hydrogels without ionic crosslinking showed the opposite trend with increased swelling in the hydrogels prepared with higher Alg concentrations. To assess the stability of these hydrogels, samples of both ionically crosslinked and non-ionically crosslinked hydrogels were fully dried and their dry mass was determined. Samples of each hydrogel were also stored in deionized water (with daily changes of water) for one week, then fully dried and weighed. The difference in dry mass of the as-prepared and water-immersed samples was assumed to be due to the loss of alginate from the gel during water immersion. The amount of alginate dissolved as a proportion of the alginate in the as-prepared samples is given in Figure 3. For the non-ionically crosslinked samples, almost all of the alginate was dissolved during water immersion and between $20 \%$ - 40\% of Alg was removed from PAAm-Alg hydrogels that were first ionically crosslinked. The implication of these observations is that the non-crosslinked Alg can be readily removed from the PAAm network by dissolution and there is no stable chemical coupling between the Alg and PAAm (in contrast to as recently claimed ${ }^{13}$ ). Ionic crosslinking of the Alg improves its retention within the PAAm network, however, a large amount of the biopolymer can still be removed for the level of ionic crosslinking used in the present study. 


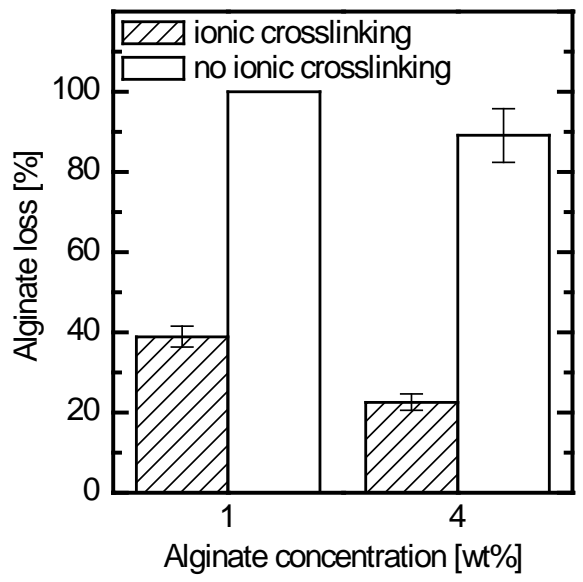

Figure 3 Alginate loss during immersion in water for one week from PAAm-based hydrogels that were initially ionically crosslinked or prepared with no ionic crosslinking .

The equilibrium swelling ratios reflect the crosslink density formed in the hydrogels. Increasing the amount of either chemical or ionic crosslinking by addition of higher concentrations of MBAA or alginate, respectively, reduces the equilibrium swelling. For the non-ionically crosslinked hydrogels, the swelling in water removes almost all of the Alg so the swelling ratio reflects the topography of the PAAm or PAA covalently crosslinked network. The swelling of the hydrogels increased when higher Alg concentrations were used and the hydrogels were not ionically crosslinked. Alginate increases significantly the viscosity of the monomer solution and the decreased molecular mobility likely reduces the number of covalent crosslinks that can form during the free radical polymerization of AA or AAm. It is important to note that the reduced covalent crosslinking also occurs in the ionicallycrosslinked hydrogels, although the ionic crosslinking reduces the equilibrium swelling and masks the increased swellability of the covalent network.

\section{Tensile Mechanical Properties}

Young's moduli and tensile strengths of PAAm-based hydrogels are presented in Figures 4 and 5 as a function of Alg concentration in the polymerization solution. A steady increase in the Young's modulus and tensile strength was observed for the ionically crosslinked hydrogels as the Alg concentration increased (Figures $4 \mathrm{a}$ and $5 \mathrm{a}$ ). In contrast, the moduli and strength of the non-ionically crosslinked hydrogels decreased with increasing Alg content (Figures $4 \mathrm{~b}$ and $5 \mathrm{~b}$ ). The decrease was most noticeable for the tensile strength. The strengths and moduli of the non-ionically crosslinked hydrogels were considerably smaller than the equivalent ionically crosslinked gels. A small increase in modulus and strength was produced when the chemical crosslinker concentration was increased at all Alg levels and for both ionic and non-ionic crosslinking. 

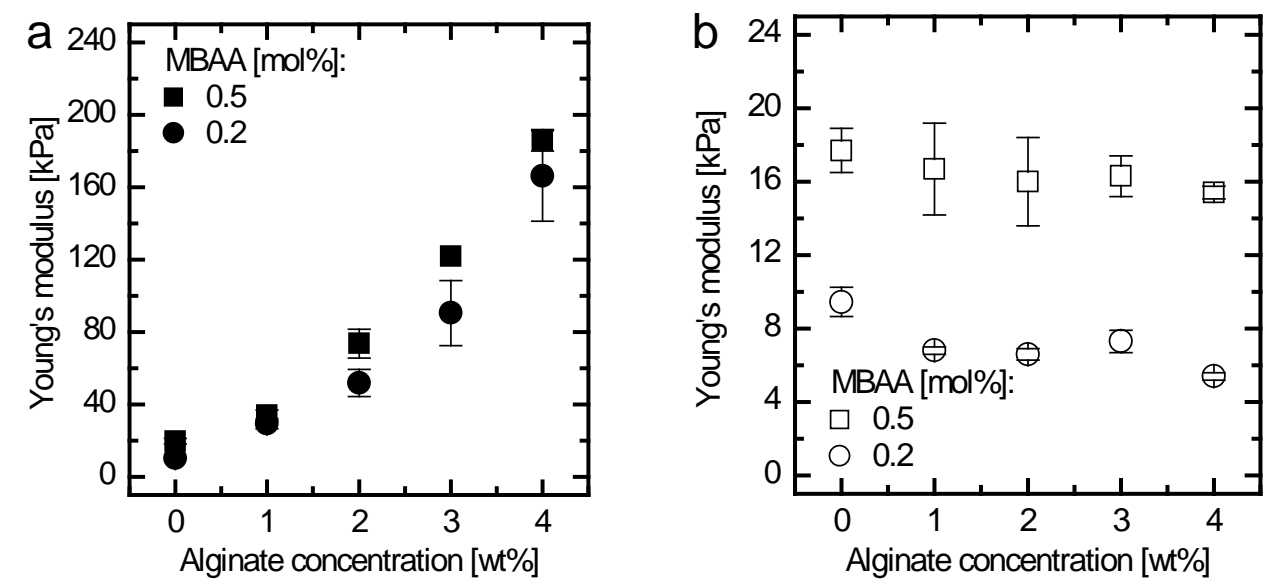

Figure 4 Young's modulus of (a) ionically crosslinked PAAm-Alg IPN hydrogels and (b) PAAmbased hydrogels with no ionic crosslinking. Alginate concentration represents the initial concentration of Alg in the polymerization solution. Note different $y$-axis scales are used in each figure.
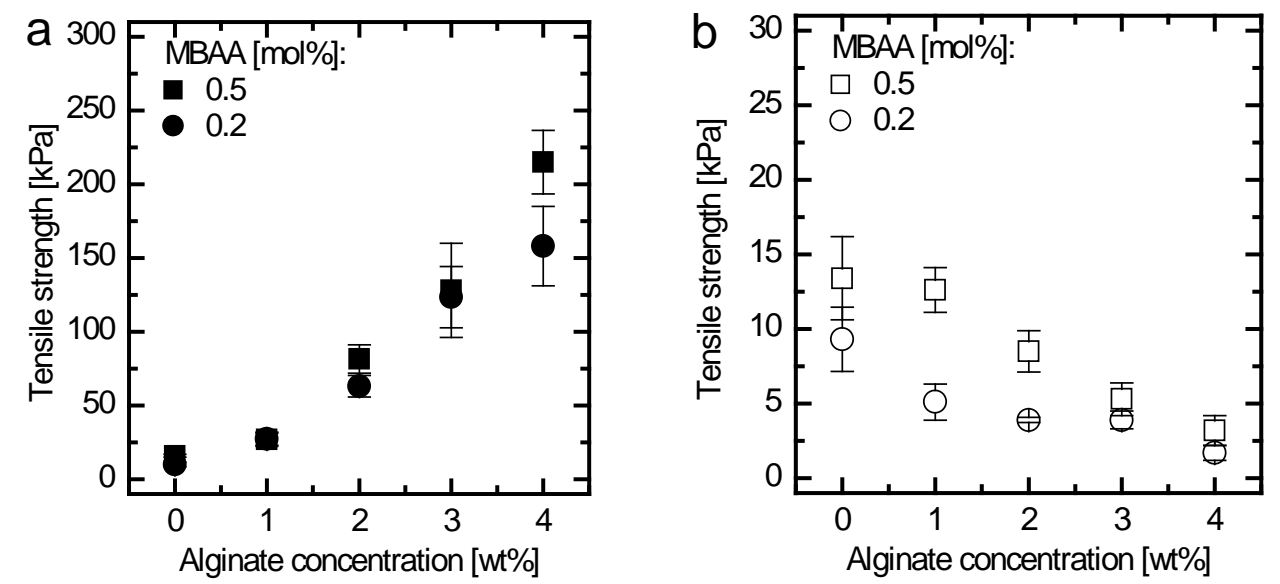

Figure 5 Tensile strength of (a) ionically crosslinked PAAm-Alg IPN hydrogels and (b) PAAm-based hydrogels with no ionic crosslinking. Alginate concentration represents the initial concentration of Alg in the polymerization solution. Note different y-axis scales are used in each figure. 

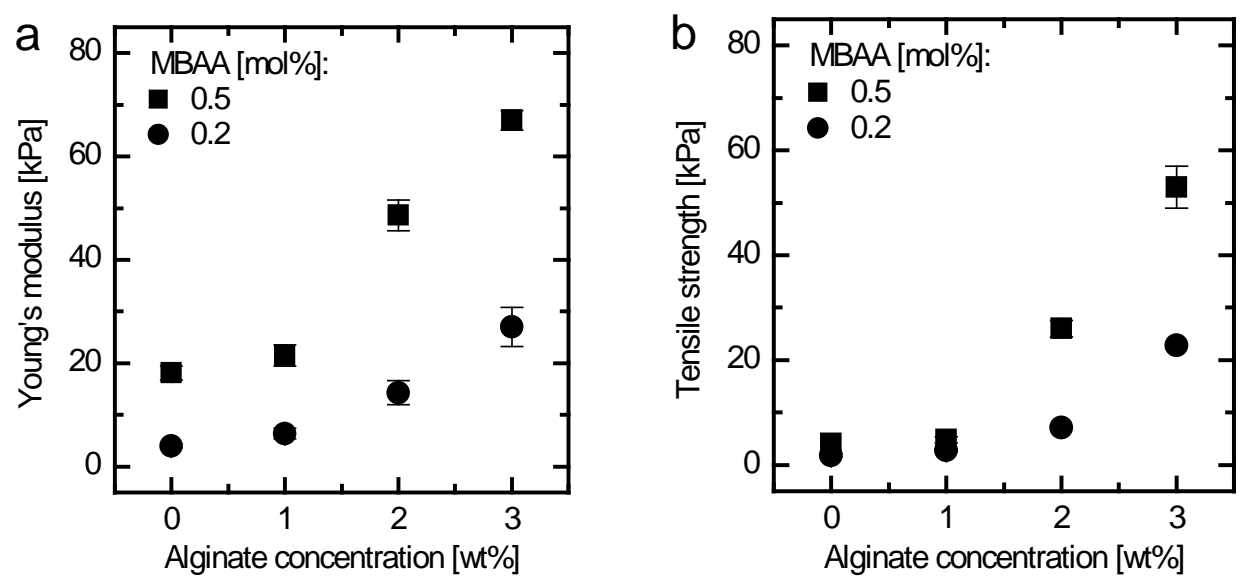

Figure 6 Tensile properties of ionically crosslinked PAA-Alg IPN hydrogels: (a) Young's modulus, (b) tensile strength. Alginate concentration represents the initial concentration of Alg in the polymerization solution.

Similar trends in tensile mechanical properties were also observed in ionically crosslinked PAA-Alg IPN hydrogels prepared with various Alg concentrations (Figure 6). PAA-based hydrogels without ionic crosslinking were extremely fragile and unsuitable for tensile testing. Both the Young's modulus and tensile strength of ionically crosslinked PAA-Alg hydrogels increased with increasing Alg content and with higher degree of chemical crosslinking. When compared with PAAm-Alg hydrogels, the PAA-Alg hydrogels had considerably smaller tensile strength and Young's modulus. The Young's modulus of ionically crosslinked PAAm-Alg IPN hydrogels was $2-6$ times larger than that of PAAAlg IPN hydrogels, and their tensile strength was $4-7$ times larger than that of PAA-Alg IPN hydrogels.

\section{Indentation Testing}

Stiffness of PAAm- and PAA-based hydrogels was also investigated using an indentation method. An indentation test can be used to measure the local modulus of a non-uniform material such as the gradient hydrogel of interest here. Indentation testing is also useful in characterising the modulus of gels that are too brittle for tensile testing. It is important to note that when an indenter is pressed on a fully immersed gel the recorded force is time-dependant as the indentation pressure causes a readjustment in the equilibrium water content. ${ }^{28}$ The stress relaxation, however, has an exponential correlation with time and is not significant at short times. ${ }^{29}$ Here, the experiments were performed in air with the maximum indentation depth of $100 \mu \mathrm{m}$ at the indentation speed of $0.1 \mathrm{~mm} / \mathrm{min}$. Thus, the total experimental time scale is $60 \mathrm{sec}$, which remains short enough to avoid any stress relaxation. ${ }^{24}$ No stress relaxation was observed during the course of indentation experiments. 

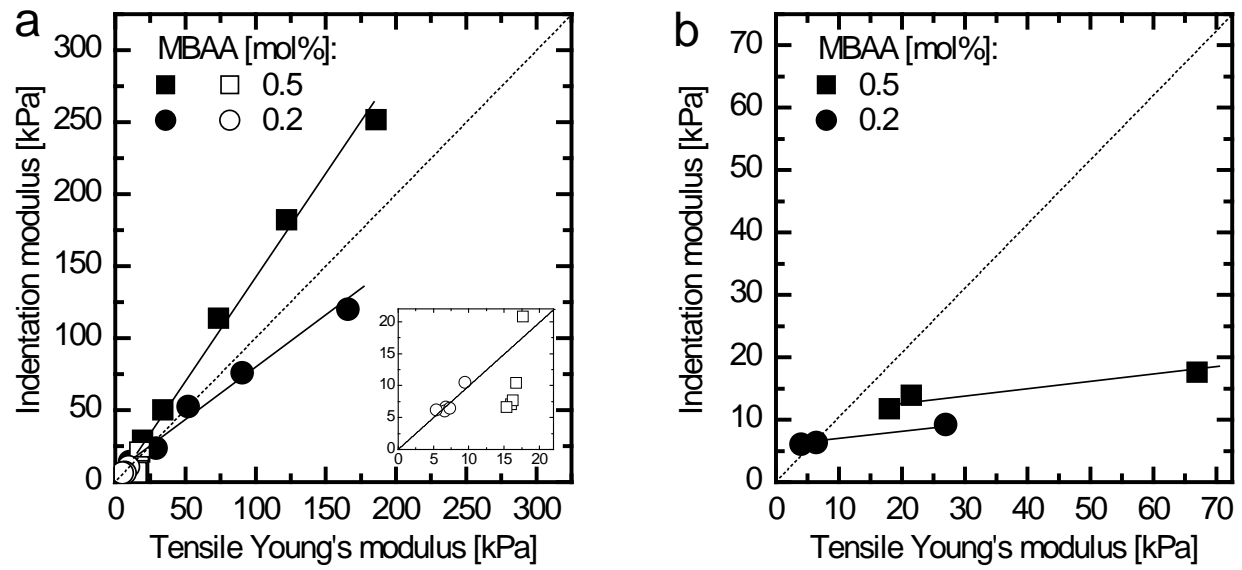

Figure 7 Modulus determined by indentation testing on (a) PAAm-based hydrogels, and (b) PAAbased hydrogels. Ionically crosslinked hydrogels are represented by filled symbols, and hydrogels with no ionic crosslinking are represented by open symbols. The level of covalent crosslinker (MBAA) is as indicated. Solid lines are the best linear fit, and dotted lines indicate the equal indentation and tensile moduli.

The moduli obtained from the indentation test (equation 3) of hydrogels with different amounts of Alg are compared with the Young's modulus obtained from the tensile test (Figure 7). The moduli obtained from the indentation test were of the same order of magnitude as the Young's moduli obtained from the tensile test. Both tests showed the same trends in terms of the effects of Alg content and level of crosslinking (ionic and covalent) on the moduli. In general, the agreement between indentation and tensile moduli for PAAm-based hydrogels is considered to be very good (with the exception of those with no ionic crosslinking and 0.5 mol\% MBAA) considering the difficulties in measuring strain accurately in a tensile test. However, the indentation moduli of PAA-Alg IPN hydrogels were considerably smaller than the tensile moduli. Disagreements between modulus results from tensile and indentation tests have been reported previously for biological tissues with orders of magnitude deviations ( $\mathrm{kPa}$ in indentation verses MPa in tensile) ${ }^{30}$ These large deviations were attributed to the fact that most of biological tissues are not homogeneous entities and indentation test probe local mechanical properties while the bulk properties are being measured in a tensile test. ${ }^{25}$ The IPN samples prepared here were macroscopically uniform, so it remains unknown as to reasons for the large discrepancy in moduli obtained for the PAA-Alg hydrogels. Not shown in Figure 7 are the moduli of PAA-based hydrogels prepared without ionic crosslinking, since tensile testing was not possible with these brittle samples. Indentation tests were performed on these hydrogels giving moduli of $11-13 \mathrm{kPa}$ and $~ 5 \mathrm{kPa}$ for $0.5 \mathrm{~mol} \% \mathrm{MBAA}$ and $0.2 \mathrm{~mol} \%$ MBAA, respectively, and regardless of the concentration of Alg used.

\section{Comparison of Moduli and Swelling Ratios}

It is intriguing to note that rather small additions of Alg can produce major changes in the network structure and mechanical properties. To more clarify the nature of the network topologies, the Young's modulus of neutral PAAm-based hydrogels are shown in Figure 8 plotted against the hydrogel swelling ratios. Two distinct regimes can be seen in Figure 8. The Young's modulus of ionically crosslinked PAAm-Alg IPN hydrogels is much more sensitive to the swelling ratio 
compared to the PAAm-based hydrogels prepared without ionic crosslinking. The power-law exponent for ionically crosslinked PAAm-Alg IPN hydrogels is, respectively, -2.65 and -2.35 for $0.5 \mathrm{~mol} \%$ and $0.2 \mathrm{~mol} \% \mathrm{MBAA}$. In the classic rubber elasticity theory ${ }^{31}$ the predicted power-law exponent for the modulus of a swollen polymer network versus swelling ratio is -3 for a $\theta$ solvent and -2.3 for an athermal solvent (see Supporting Information). The experimentally determined Young's modulus and swelling ratio for the ionically crosslinked PAAm-Alg IPN hydrogels are close to these theoretical values.

In contrast, the moduli of non-ionically crosslinked PAAm-based hydrogels were relatively insensitive to the swelling ratio. The power-law exponents were -0.08 or -0.33 for the two different MBAA crosslinking ratios. As described above, the Alg is almost completely removed from the nonionically crosslinked hydrogels during equilibration in water. The resultant equilibrated networks are then simply PAAm single networks swollen to equilibrium with water and the equilibrium modulus reflects the extent of dilution and chain extension. The equilibrium swelling ratios of these single networks are much higher than an equivalent network with ionically crosslinked Alg (Figure 1). Increasingly higher swelling ratios eventually cause the network chains to become fully extended, so that the classical rubber elasticity theory no longer applies. The result of the chain extension in these highly swollen PAAm single networks is higher than expected moduli. ${ }^{32}$

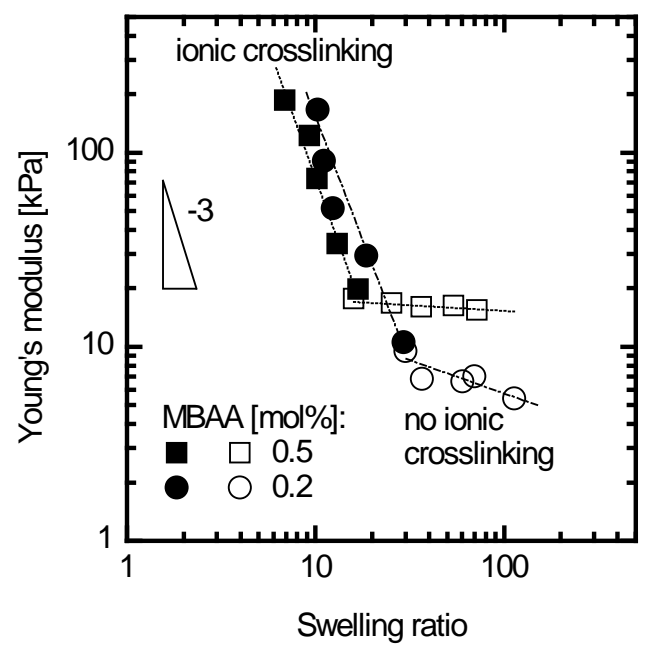

Figure 8 Young's modulus of PAAm-based hydrogels as a function of equilibrium swelling ratio, for ionically crosslinked IPN hydrogels (filled) and hydrogels with no ionic crosslinking (open). Lines are the best power-law fit. The -3 slope is indicated from rubber elasticity theory for a swollen network in a $\theta$ solvent.

\section{Fracture Toughness}

It has recently been reported that PAAm-Alg hydrogels can show remarkably high toughness. ${ }^{13}$ The recent work of Sun et al. considered only the toughness of as-prepared gels and it is not known if the high toughnesses reported by these workers are retained at equilibrium swelling. Here we report toughnesses for PAAm-Alg hydrogels prepared with different amounts of MBAA chemical crosslinker and Alg contents and swollen to equilibrium in deionized water. As shown in Figure 9a, the toughness of ionically crosslinked PAAm-Alg IPN hydrogels decreased monotonically as the MBAA concentration increased. The toughest hydrogels with only $0.2 \mathrm{~mol} \% \mathrm{MBAA}$ in the polymerization solution had a fracture energy of $217 \pm 11 \mathrm{~J} / \mathrm{m}^{2}$. In contrast, hydrogels with $2 \mathrm{~mol} \%$ 
MBAA had a fracture energy of $27 \pm 6 \mathrm{~J} / \mathrm{m}^{2}$. Sun et al. reported a peak in toughness at $0.06 \mathrm{wt} \%$ MBAA ( 0.027 mol\% based on monomer) when the MBAA content was increased from $0.03 \mathrm{wt} \%$ ( $0.014 \mathrm{~mol} \%)$ to $0.12 \mathrm{wt} \%(\sim 0.055 \mathrm{~mol} \%){ }^{13}$ In IPN hydrogels consisting of two interpenetrating covalent networks (so called "double network hydrogels") where a loosely crosslinked second network is formed within a tighter first network, the toughness also passes through a maximum as the crosslink density of the loose second network decreases. ${ }^{17}$ The ionic-covalent IPN hydrogels investigated here consist of a tighter ionically crosslinked Alg network and a more loosely crosslinked PAAm network. The increase in toughness of these hydrogels with decreasing crosslink density of the PAAm network is consistent with the results presented previously for double network hydrogels and similar PAAm-Alg hydrogels prepared with a wider range of MBAA concentrations.

The fracture energy of the PAAm-Alg hydrogels (0.5 mol\% MBAA) increased with increasing Alg content (Figure 9b). Sun et al. reported a peak in toughness at a weight ratio of PAAm to Alg of 5.6 for similar hydrogels tested without swelling to equilibrium. ${ }^{13}$ The AAm:Alg ratio of hydrogels investigated in the present study decreased from 20 (1 wt\% Alg) to $3-4$ (4 wt\% Alg), when Alg loss was taken into account. The fracture energy of ionically crosslinked PAAm-Alg IPN hydrogels increased from $9 \pm 2 \mathrm{~J} / \mathrm{m}^{2}$ (AAm:Alg 20) to $70 \pm 2 \mathrm{~J} / \mathrm{m}^{2}$ (AAm:Alg $~ 3-4$ ) in general agreement with the observations by Sun et al. In the covalent double network hydrogels ${ }^{9}$ the toughness increases rapidly with an increasing ratio of second (loose) network to the first (tighter) network and then levels off at higher ratios (mole ratio > 10). A different trend is observed in the ionic-covalent IPN hydrogels with a peak in toughness occurring with increasing ratio of loose (Alg) to tight (PAAm) network. The reasons for these differences are not clear, although it is known that covalent connections typically occur between the two covalent networks as a result of unreacted functional groups of the first network participating in the second network formation (unless the remaining unreacted groups are deliberately removed prior to the second network polymerization ${ }^{17}$ ). Reaction between the Alg and PAAm networks prepared here does not occur since the Alg can be easily extracted if it is not treated with $\mathrm{CaCl}_{2}$.
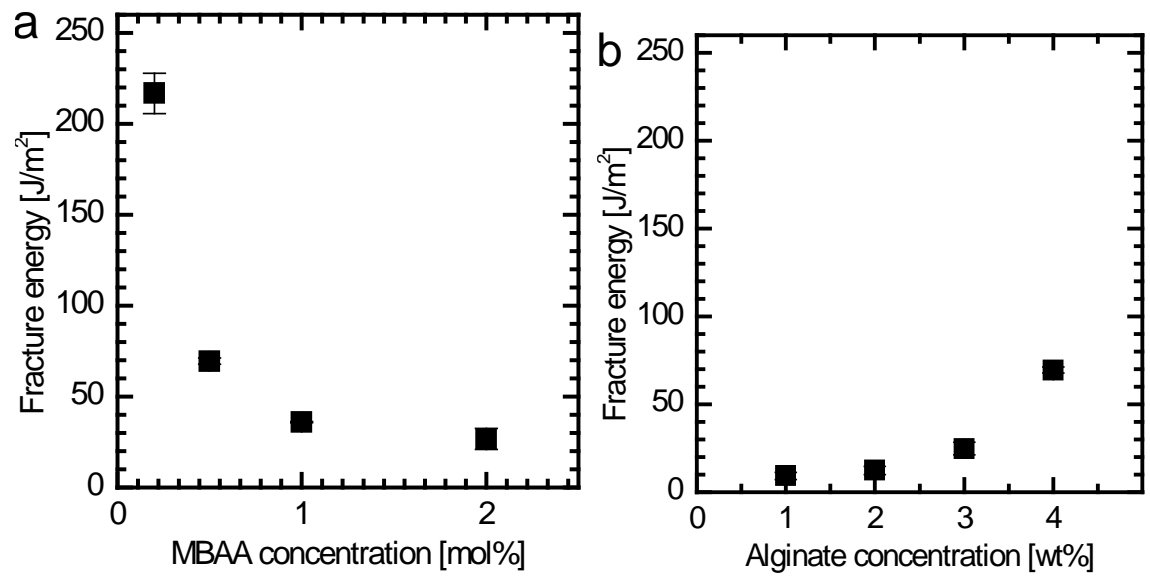

Figure 9 Fracture energy of ionically crosslinked PAAm-Alg IPN hydrogels as a function of (a) MBAA, and (b) Alg concentration in the polymerization solution. Alg concentration in (a) is $4 \mathrm{wt} \%$ and MBAA concentration in (b) is $0.5 \mathrm{~mol} \%$. 


\section{Gradient Gels as Artificial Tendons}

The ability to control gel stiffness and swelling while retaining reasonable toughness is useful for preparing gel-based artificial muscles. Where the hydrogel is connected to a more rigid structure, the mismatch in volume generates considerable stress at the interface ${ }^{33}$ that can cause delamination or fracture. Nature solves this problem by connecting muscle to bone with tendons. The tendons have a stiffness that is intermediate between the soft muscle $(\sim 1.5-3.2 \mathrm{kPa})^{34}$ and the hard bone $(\sim 20-30$ $\mathrm{GPa})$ and a gradient in mechanical properties along their length. ${ }^{35}$

Here, we use the ionic-covalent IPN hydrogel materials to produce hydrogel 'tendons' for connecting a hydrogel artificial muscle to its rigid frame. A gradient in mechanical properties and swellability was introduced by sequentially adding and partially polymerizing layers of PAAm-Alg or PAA-Alg hydrogel with the desired properties (Figure 10). The highly swellable and $\mathrm{pH}$ sensitive middle section of the sample consisted of PAA-Alg with $4 \mathrm{wt} \%$ Alg. Both ends of the sample were prepared from 4 layers of PAAm-Alg with the Alg content decreasing from $4 \mathrm{wt} \%$ to $1 \mathrm{wt} \%$ from the ends towards the centre. As expected, the PAA containing middle section of the gel showed considerable volume and modulus change in response to the surrounding $\mathrm{pH}$ (Figure 11). The gel ends, however, were insensitive to $\mathrm{pH}$. These tendon-like ends can be used to make stable mechanical connections to a rigid frame.

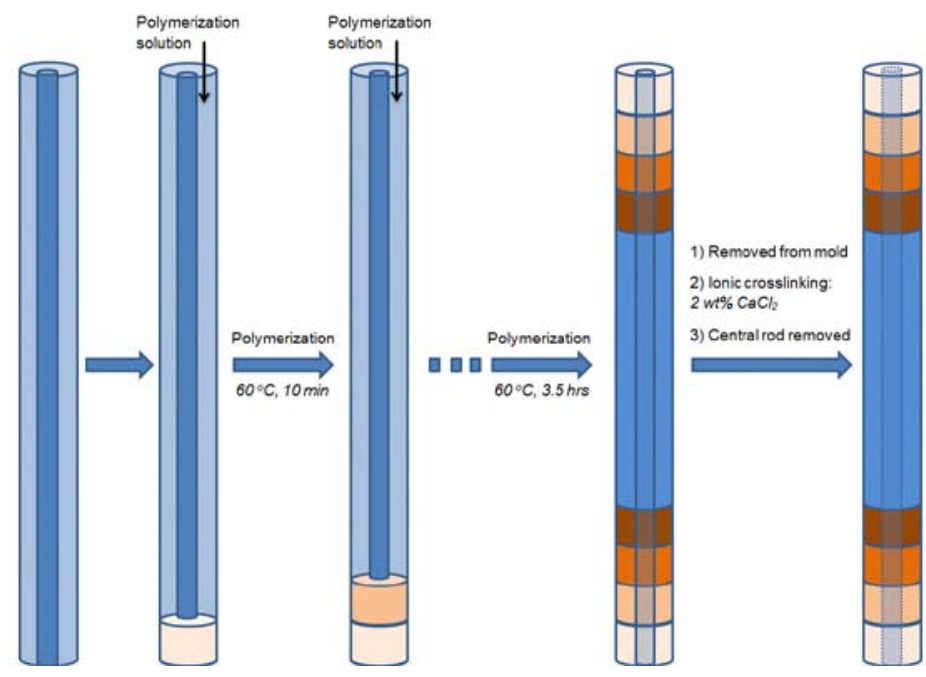

Figure 10 Schematic illustration of the process of making a hollow cylindrical hydrogel with gradients in its structure. Each small section is made of a PAAm-Alg IPN with different amount of $\mathrm{Alg}$, and the larger section in the middle is the pH-sensitive part made of PAA-Alg. 


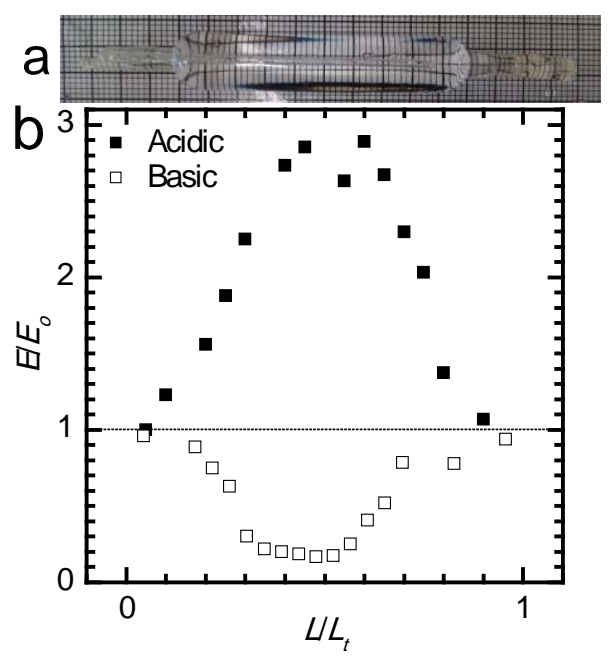

Figure 11 (a) Photograph of a hollow cylinder gradient hydrogel after equilibrating at $\mathrm{pH} 10$. (b) Indentation modulus normalised to the modulus measured in deionized water as measured at various locations along a flat sheet of hydrogel prepared with spatially different PAAm-Alg or PAA-Alg contents.

\section{CONCLUSIONS}

PAAm-Alg and PAA-Alg IPN hydrogels were developed by radical polymerization and covalent crosslinking of, respectively, AAm and AA monomers in the presence of Alg. Ionic crosslinking of Alg was performed by storing the as-prepared PAAm-Alg and PAA-Alg hydrogels in $\mathrm{CaCl}_{2}$ solution. PAAm- and PAA-based hydrogels with no ionic crosslinking were also prepared for comparison. Increasing the Alg content of ionically crosslinked PAAm-Alg and PAA-Alg IPN hydrogels considerably enhanced their Young's modulus, tensile strength, indentation modulus, and fracture energy. However, without ionic crosslinking a higher concentration of Alg in the polymerization solution resulted in reduced modulus, strength and toughness and increased swelling ratio. Without ionic crosslinking more than $90 \%$ of Alg that was added to the polymerization solution was dissolved during immersion in water. It was apparent that the presence of Alg chains during the covalent network formation permanently changed the network structure of PAAm and PAA. Hence, the physical and mechanical properties of PAAm- and PAA-based hydrogels (both with and without ionic crosslinking) were influenced by the Alg concentration used in their polymerization solutions. We also demonstated that the fracture energy decreased with increasing degree of covalent crosslinking and increased with increasing Alg concentration when ionic crosslinking was performed. Toughness values for the hybrid systems described here are similar to previously described tough gels of similar swelling ratio and modulus. A gradient hydrogel was made with a hollow channel in centre. The middle part of the hydrogel was $\mathrm{pH}$-sensitive while the rest of the hydrogel was insensitive to $\mathrm{pH}$. This structure can be useful in the field of sensors and soft actuators where a structural gradient is required to connect a stimulus-sensitive hydrogel to more rigid substrates. 


\section{ACKNOWLEDGEMENTS}

The authors acknowledge the Australian Research Council for financial support through its Centre of Excellence and Professorial Fellowship schemes.

\section{REFERENCES}

1. Hoffman, A. S. Adv. Drug Delivery Rev. 2002, 43, 3.

2. Harmon, M. E.; Tang, M.; Frank, C. W. Polymer 2003, 44, 4547.

3. Moschou, E. A.; Peteu, S. F.; Bachas, L. G.; Madou, M. J.; Daunert, S. Chem. Mater. 2004, 16, 2499.

4. Kwon, G. H.; Choi, Y. Y.; Park, J. Y.; Woo, D. H.; Lee, K. B.; Kim, J. H.; Lee, S. -H. Lab Chip 2010, 10, 1604.

5. Zhang, X.; Pint, C. L.; Lee, M. H.; Schubert, B. E.; Jamshidi, A.; Takei, K.; Ko, H.; Gillies, A.; Bardhan, R.; Urban, J. J.; Wu, M.; Fearing, R.; Javey, A. Nano Lett. 2011, 11, 3239.

6. Naficy, S.; Brown, H. R.; Razal, J. M.; Spinks, G. M.; Whitten, P. G. Aust. J. Chem. 2011, 64, 1007.

7. Okumura, Y.; Ito, K. Adv. Mater. 2001, 13, 485.

8. Haraguchi, K.; Takehisa, T. Adv. Mater. 2002, 14, 1120.

9. Gong, J. P.; Katsuyama, Y.; Kurokawa, T.; Osada, Y. Adv. Mater. 2003, 15, 1155.

10. Huang, T.; Xu, H.; Jiao, K.; Zhu, L.; Brown, H. R.; Wang, H. Adv. Mater. 2007, 19, 1622.

11. Malkoch, M.; Vestberg, R.; Gupta, N.; Mespouille, L.; Dubois, P.; Mason, A. F.; Hedrick, J. L.; Liao, Q.; Frank, C. W.; Kingsbury, K.; Hawker, C. J. Chem. Commun. 2006, 2774.

12. Sakai, T.; Matsunaga, T.; Yamamoto, Y.; Ito, C.; Yoshida, R.; Suzuki, S.; Sasaki, N.; Shibayama, M.; Chung, U. -i Macromolecules 2008, 41, 5379.

13. Sun, J. -Y.; Zhao, X.; Illeperuma, W. R. K.; Chaudhuri, O.; Oh, K. H.; Mooney, D. J.; Vlassak, J. J.; Suo, Z. Nature 2012, 486, 133.

14. Myung, D.; Waters, D.; Wiseman, M.; Duhamel, P.-E.; Noolandi, J.; Ta, C. N.; Frank, C. W. Polym. Adv. Technol. 2008, 19, 647.

15. Waters, D. J.; Engberg, K.; Parke-Houben, R.; Ta, C. N.; Jackson, A. J.; Toney, M. F.; Frank, C. W. Macromolecules 2011, 44, 5776.

16. Tanaka, Y.; Kuwabara, R.; Na, Y. -H.; Kurokawa, T.; Gong, J. P.; Osada, Y. J. Phys. Chem. B 2005, 109, 11559.

17. Nakajima, T.; Furukawa, H.; Tanaka, Y.; Kurokawa, T.; Osada, Y.; Gong, J. P. Macromolecules 2009, 42, 2184.

18. Yu, Q. M.; Tanaka, Y.; Furukawa, H.; Kurokawa, T.; Gong, J. P. Macromolecules 2009, 42, 3852.

19. Lee, Y. M.; Lee, K. H.; Kim, S. J. J. Appl. Polym. Sci. 1999, 73, 113.

20. Ju, H. K.; Kim, S. Y.; Lee, Y. M. Polymer 2001, 42, 6851.

21. Sun, J.; Xiao, W.; Tang, Y.; Li, K.; Fan, H. Soft Matter 2012, 8, 2398.

22. Bakarich, S. E.; Pidcock, G. C.; Balding, P.; Stevens, L.; Calvert, P.; in het Panhuis, M. Soft Matter 2012, 8, 9985.

23. Lee, K. Y.; Mooney, D. J. Prog. Polym. Sci. 2012, 37, 106.

24. Kuo, C. K.; Ma, P. X. Biomaterials 2001, 22, 511. 
25. Perrin, D. D.; Dempsey, B.; Serjeant, E. P. In pKa Prediction for Organic Acids and Bases; Chapman \& Hall: London, 1981.

26. Ulman, A. In An Introduction to Ultrathin Organic Films; Academic Press: San Diego, 1991.

27. Johnson, K. L. In Contact Mechanics; Cambridge University Press: Cambridge, 1985.

28. Hui, C. -Y.; Lin, Y. Y.; Chuang, F. -C.; Shull, K. R.; Lin, W. -C. J. Polym. Sci. B 2006, 43, 359.

29. Hu, Y.; Zhao, X.; Vlassak, J. J.; Suo, Z. Appl. Phys. Lett. 2010, 96, 121904.

30. McKee, C. T.; Last, J. A.; Russell, P.; Murphy, C. J. Tissue Eng. B 2011, 17, 155.

31. Treloar, L. R. G. In The Physics of Rubber Elasticity; Oxford University Press: Oxford, 1975.

32. Dubrovskii, S. A. Polym Gels Netw. 1996, 4, 467.

33. Kang, M. K.; Huang, R. J. Appl. Mech. 2010, 77, 061004.

34. Chen, E. J.; Novakofski, J.; Jenkins, W. K.; O’Brien, W. D. IEEE Trans. Ultrason. Ferroelectr. Freq. Control 1996, 43, 191.

35. Genin, G. M.; Kent, A.; Birman, V.; Wopenka, B.; Pasteris, J. D.; Marquez, P. J.; Thomopoulos, S. Biophys. J. 2009, 97, 976. 